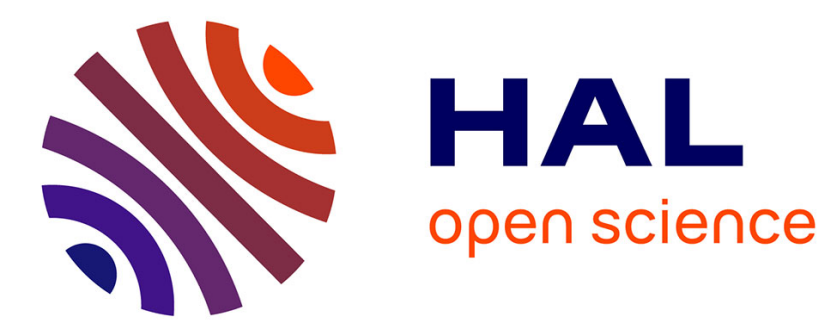

\title{
Study on the Utilization of Digital Manufacturing for Production Operations
}

\author{
Masahiro Shibuya, Kenichi Iida, Koki Mikami
}

\section{To cite this version:}

Masahiro Shibuya, Kenichi Iida, Koki Mikami. Study on the Utilization of Digital Manufacturing for Production Operations. IFIP International Conference on Advances in Production Management Systems (APMS), Aug 2018, Seoul, South Korea. pp.139-145, 10.1007/978-3-319-99707-0_18 . hal02177885

\section{HAL Id: hal-02177885 \\ https://hal.inria.fr/hal-02177885}

Submitted on 9 Jul 2019

HAL is a multi-disciplinary open access archive for the deposit and dissemination of scientific research documents, whether they are published or not. The documents may come from teaching and research institutions in France or abroad, or from public or private research centers.
L'archive ouverte pluridisciplinaire HAL, est destinée au dépôt et à la diffusion de documents scientifiques de niveau recherche, publiés ou non, émanant des établissements d'enseignement et de recherche français ou étrangers, des laboratoires publics ou privés. 


\title{
Study on the utilization of digital manufacturing for production operations
}

\author{
Masahiro Shibuya $^{1}$, Kenichi Iida ${ }^{2}$ and Koki Mikami ${ }^{3}$ \\ ${ }^{1}$ Tokyo Metropolitan University, 6-6 Asahigaoka, Hino, Tokyo, JAPAN \\ ${ }^{2}$ Hokkaido Industrial Research Institute, kita19 nishi11, kita-ku, Sapporo, JAPAN \\ ${ }^{3}$ Hokkaido University of Science, 4-1, Maeda 7-jo 15-chome, Teine-ku, Sapporo, JAPAN \\ mshibuya@tmu.ac.jp
}

\begin{abstract}
Digital manufacturing (DM), which has developed rapidly in recent years, is premised on use in planning operations, and it is not utilized in production operation. There are many challenges to the application of DM in production operations, such as the incapacity of a supervisor at a work site to effectively implement the manufacturing-line model developed using a DM, in a planning operation. Therefore, we propose a system that makes it possible to redesign work plans and support improvement activity by simply inputting values in accustomed standardized work tables, namely, process capacity tables, standardized work tables, and standardized work combination tables, of three types of workers, instead of inputting a large quantity of complicated parameters. Less examination time is desirable at production sites, and we added a tool that enabled the graphical display of execution processes and results. Moreover, we added a system that made it possible to alter Microsoft Excel database inputs, such as the number or arrangement of DM processing machines. This paper describes the proposed system and its method of implementation.
\end{abstract}

Keywords: Digital Manufacturing, 3 kinds of standardized work tables, improvement operation.

\section{Introduction}

Technical developments for the realization of a "Smart Factory," which is an advanced factory substantiating the different manufacturing strategies (e.g., Industry 4.0, industrial Internet, cloud manufacturing) proposed by various countries, is flourishing. IEC 62832 (Digital Factory DF), which is aimed at the integrated management of physical and virtual spaces by digitizing the whole factory, is advocated. The method, called Digital Twin, was proposed by Grieves in 2003. Based on this idea, a concept is being developed to reproduce the physical manufacturing space in real time by digitization and to make the control and management of real factories easier. In German Industry 4.0, the optimization of all processes related to the manufacturing industry by CPS (Cypher Physical System) is underway. Some large enterprises (e.g., Siemens, Dassault, and PTC) use Digital Twin to support their customer services with state-of-the-art technology (e.g., aerospace technology for aircraft maintenance, etc.). 
Tao classified the factory evolution process to achieve coalescence of physical and virtual spaces into four stages. Digital Twin is the fourth stage; the present is the third stage, in which physical and virtual spaces interact with each other. What is imperative in the third stage is production technique support using 3D CAD called Digital Manufacturing (DM). This method was developed to support the shortening of development periods by front-loading activities from product development by concurrent engineering to production preparation and process mounting.

Digital Manufacturing (DM), which has rapidly developed in recent years, is premised on its application in "planning operation," and will not be utilized in "production operation." To utilize DM in "production operation," the supervisor at a work site must be able to understand the manufacturing line model made by DM and possess the ability to modify it for factory improvement. Many experts say that manufacturing line simulators are difficult to master and are now experts' tools. In the Japanese manufacturing industry, the structure in which workers independently advance toward the achievement of their goals using various kinds of management tables of their own and repeating the PDCA cycle is widely used.

Therefore, we first examined how to utilize DM at a production site, rather than aiming for Digital Twin directly. For small and medium-sized enterprises unfamiliar with ICT, we thought it necessary to develop a mechanism that supports production redesign and improvement activities by inputting values into three kinds of workers' accustomed standardized work tables (process capacity tables, standardized work tables, and standardized combination tables) with click of a button, instead of inputting a large quantity of complex parameters. In this mechanism, based on the production plan made at an office, a virtual line is constructed for DM, and evaluation and improvement are conducted. Our aim is the development of a mechanism which makes it possible to seamlessly transfer the values in the process capacity table to the parameters of machines, such as the processing machines on the virtual line. The user can make the best standardized work combination table just by clicking a button. In the next step, we aim to utilize this system by feedbacking the actual operation and production information to the virtual production model, re-evaluating it, and connecting the results to factories in operation.

The purpose of this study is to create a mechanism which makes it possible to modify the original production plan (proposal) to obtain the best one suited for the manufacturing site using DM, and utilizing it effectively in the PDCA cycle.

\section{Production management problems in terms of production sites}

\subsection{How to conduct production planning and management}

In some enterprises, the production management department of the corporate office makes the production plans, and the factory is forced to manage production according to these plans. Many of the plans made at the office side are medium schedule, and at the factory side small schedule such as when to start or finish must be made. Figure 1 
shows a scene of production management. The center of the figure shows the PDCA cycle. Because the production management department makes long-term plans, it also examines process design, line design, etc. The outline is shown in the right and left of the Plan part (PLANO0).

Based on the production plan (PLAN00) made at the office side, the factory supervisor creates the process capacity table, determines the various production efficiencies of each process, modifies the plan into a practicable one by experience, decides the tackt time, and finally makes three kinds of standardized work tables. The factory is operated on the basis of the standardized work tables. The output is separately recorded, and utilized for evaluation and improvement.

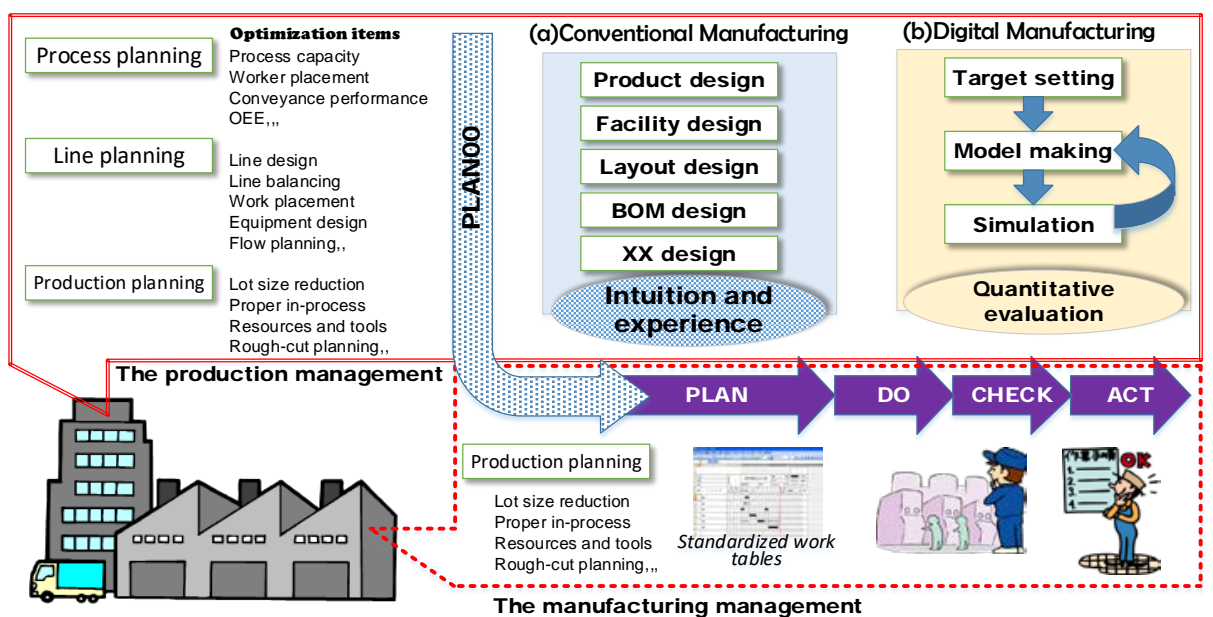

Fig. 1. Comparison between conventional and proposed production management operations.

\section{$2.2 \quad$ New production management}

Figure 1(a) shows the conventional method of starting the manufacturing of a new product. Figure 1(b) shows the method of using DM, which has become the focus of attention recently. As shown in the figure, in the stage PLAN00 of production plan management (office side), a practicable virtual line is constructed. Then, the parameters are modified to meet the target values; after repeated simulation, the new factory is quantitatively evaluated. This makes it possible to come up with an appropriate plan for the manufacturing site because the ordinary separated "process design" and "line design" are mutually connected, and to shorten the period of development and production preparation because the examination of items requiring intuition or experience, "visualization" and "optimization," can be realized in a shorter time.

Many enterprises utilize DM for the purpose of operating a highly efficient manufacturing line within a short time. DM has numerous functions, but the functions "process design," "line design," and "line operation design" are mainly utilized. The left of Figure 1 PLAN00 shows optimization items which are important in production 
planning. Examining these items one by one takes a long time from model making to evaluation; thus, it is difficult to modify the model. The range of DM use is limited because of its high cost, but with its spread, attention is now being paid on how to create an autonomous control mechanism using the analysis results of worksite data.

Some shop-floor uses of DM include workability evaluation, layout re-examination, and line balance evaluation. The primary purpose of these evaluations is identify problems or bottlenecks in lines and processes before mass production, and verifying the measures in advance. Therefore, these must be finished before the start of manufacturing. What is necessary to effectively utilize DM on the shop floor is the development of a support system that makes it possible to easily conduct "minor adjustments in production preparation," "minor adjustment by improvement," etc., using the three kinds of standardized work tables as a user interface based on the worksite supervisor's experience and intuition, and, in the years ahead, information obtained from various devices and factory observations.

\section{The summary of our system}

\subsection{The concept of the continuous improvement activity support system}

Small and medium-sized manufacturers requires quick planning of a production schedule and flexible production management for "multiproduct variable quantity production"; at the production sites, the way to effectively advance production management operations is a problem that needs to be solved. Thus, we have been researching and developing a "continuous improvement activity support system" in order to create a simple production management system whose mechanism makes it possible to collect production-site information using IoT, effectively utilize the PDCA cycle for production activities, conduct evaluation using DM, and utilize the results for re-designing production plans (Figure 2). The design enables handling of the needs of each worksite by adding necessary functions, as shown by the wheel in Figure 2.

Information inside the factory is gathered using IoT in the PDCA, as shown in the left of Figure 2, and analyzed by DM, after which minor adjustments are made for production. When the production plan is very different from the present conditions, another one is made using $\mathrm{O} 2 \mathrm{O}$ in the figure right. 


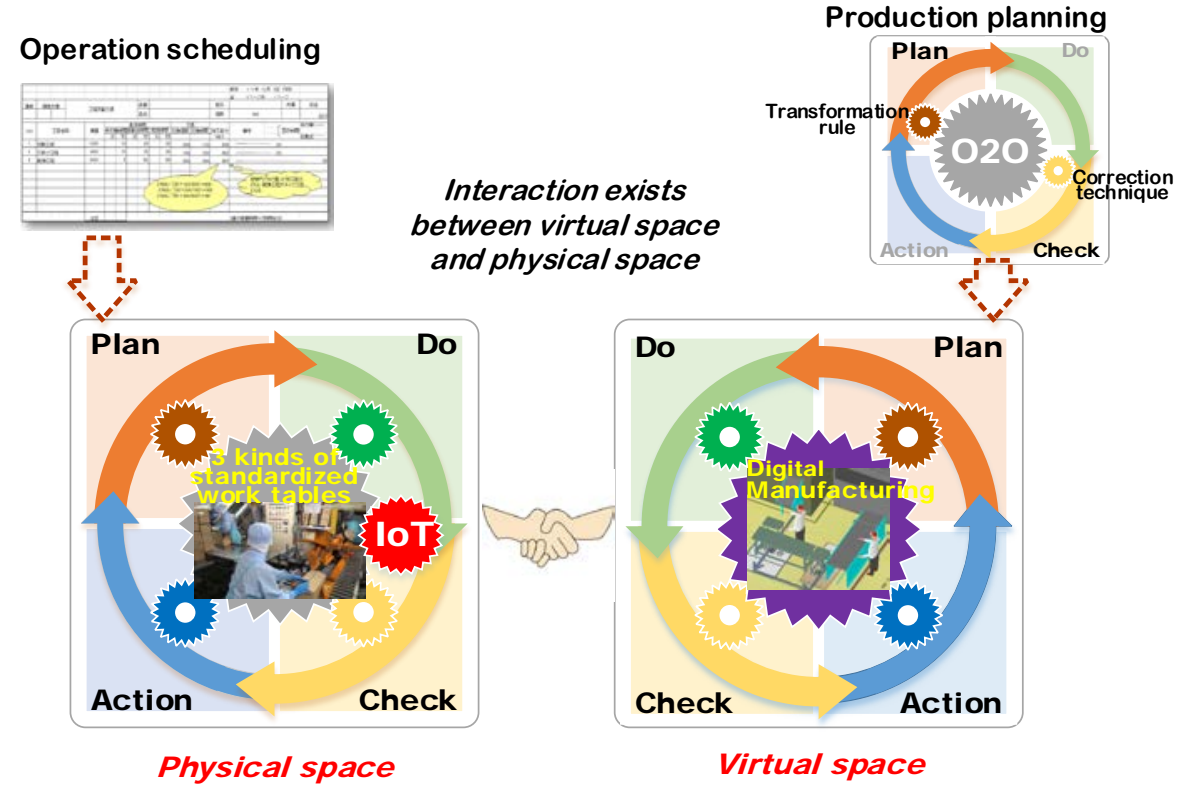

Fig. 2. Relationship between the continuous-improvement activity support system and three types of standardized work tables.

\subsection{Overview of the proposed system.}

This system stores, in the simulation model, necessary information interpreted by DM based on the process order shown in the process capacity table. Therefore, the user can conduct evaluation and examination, modify the production plan for a practicable one, and reflect the results in the PDCA cycle (see Fig. 2). The factory simulation software DELMIA Quest was used in DM. Production planning was conducted with little input for given data (items, machines, work in progress, delivery requests, etc.) and adjustment parameters, by using the online to offline (O2O) technique. The right part of Figure 2 shows a Microsoft Excel (hereby referred to as Excel) process capacity table made from the planning results.

Figure 3 shows the procedure to make a manufacturing line model using the data of standardized work tables on Excel; however, on Quest, this model can be concisely made. On Excel standardized work tables, the work order, work time, connecting order of machines, work contents, and other parameters are entered as the port. Because these contents are parameters imperceptible by Quest, by making a link between the conversion system and Excel, they are converted to BCL commands, which enables Quest to perceive the contents on Excel.

The conversion system analyzes the commands converted using the BCL program and redefines the execution order and other factors to develop a Quest model. Moreover, the system is given the role of controlling Quest. The system boots Quest and generates a manufacturing line model by transferring and executing the control command. 
Quest has the function to back-end process the commands sent from the transmission system, and to front-end process the tool for both the visualization of execution results of the model and various predictions.

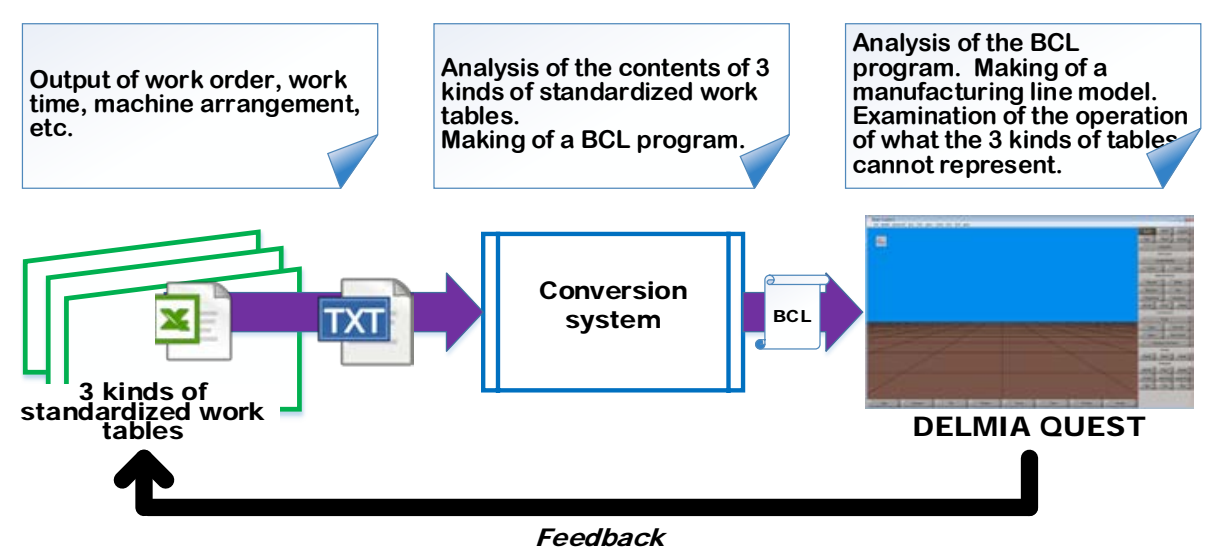

Fig. 3. Relationship between Excel, the conversion system, and Quest.

\section{How to create the manufacturing line model}

The user selects a standardized work combination table using the conversion system, and then, the system analyzes the read-in parameters for BCL command conversion. Quest analyzes and executes this control command, and a manufacturing-line model matched to the standardized work combination table is created (see Fig. 4). The conversion system judges the input contents and provides the production method logic, such as lot production, to the created model. Quest reads the particular execution time appointed by the user, and the created model automatically starts simulation. 


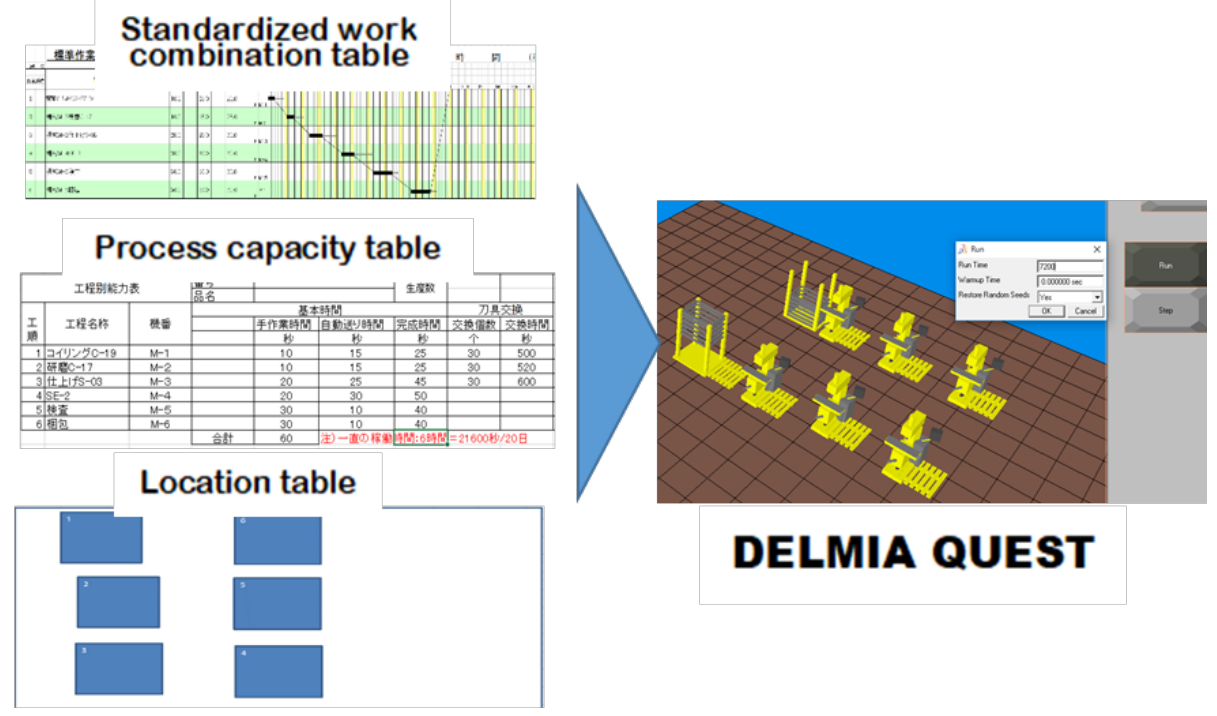

Fig. 4. Images of manufacturing-line model development using standardized work tables.

The trial system was examined at a labor-intensive factory. Our target was a part of the sheet metal processing line to manufacture parts for heating stoves. Figure 5(a) shows the layout of the target line. The rounded rectangles denote the processing machines, such as welders and press machines. The workers in this region moved and used a necessary processing machine to manufacture each part.

First, we made the three types of standardized work tables for the factory, and subsequently, we operated the system. On specifying a particular table, the model was displayed on Quest, as shown in Figure 5(b). This factory used Lot Size 1 for production. We conducted evaluation considering Lot Size 10, because our investigation noted a large occurrence of waiting periods in this zone. The examination using the visualization tool showed that two more pieces could be manufactured in the case of Lot Size 10 (Figure 5(c)).

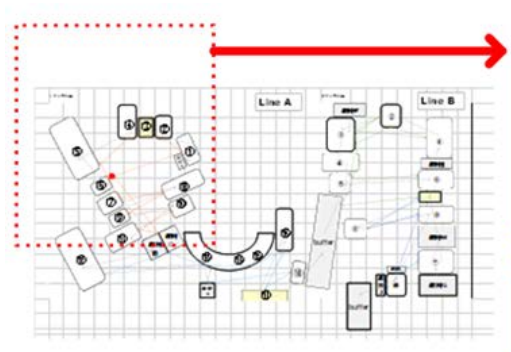

(a)

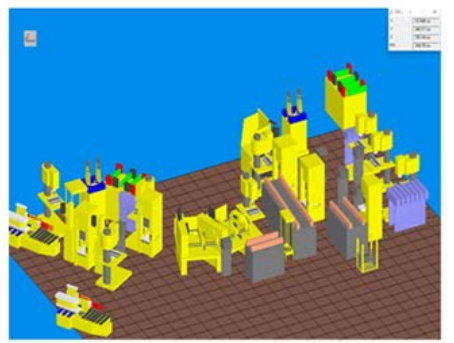

(b)

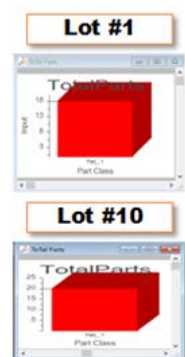

(c) 
Fig. 5. Images of the examination of the steel metal processing using our trial system

(a) Layout of the target line, (b) automatic modelling of Worker A's work position, and (c) examination using the proposed visualization tool.

\section{Conclusion}

This paper proposes a system that makes it possible to automatically generate a DM model from three types of standardized work tables. The system realized the following:

- Generation of a DM model from three types of standardized work table

- Examination of the lot production system

- $\quad$ Determination of operation rates and load ratio on the line model

- $\quad$ Storage of the standardized work tables and the line model

- $\quad$ Storage of the history of operation and examination

These results proved that this system makes it possible to modify the production plan to attain optimization by using DM at a production site. 